\title{
A Wavelet Based Method for the Solution of Fredholm Integral Equations
}

\author{
En-Bing Lin, Yousef Al-Jarrah \\ Central Michigan University, Mount Pleasant, USA \\ Email: enbing.lin@cmich.edu
}

Received December 20, 2011; revised January 17, 2012; accepted January 26, 2012

\begin{abstract}
In this article, we use scaling function interpolation method to solve linear Fredholm integral equations, and we prove a convergence theorem for the solution of Fredholm integral equations. We present two examples which have better results than others.
\end{abstract}

Keywords: Coiflet; Scaling Function Interpolation; Wavelet; Fredholm Integral Equation; Multiresolution Analysis

\section{Introduction}

Integral equations play an important role in both mathematics and other applicable areas. Many physical phenomena can be modeled by differential equations. In fact, a differential equation can be replaced by an integral equation that incorporates its boundary conditions. Integral equations are also useful in many branches of pure mathematics as well. Here we study Fredholm integral Equations [1-3].

Wavelets have been applied in a wide range of engineering and physicaldisciplines, and it is an exciting tool for mathematicians. In this paper we will find a numerical solution for the second kind Fredholm integral equation of the form

$$
y(x)=g(x)+\int_{a}^{b} k(x, t) y(t) d t,
$$

where the function $g(x)$ and are $k(x, t)$ given, and the unknown function $y(t)$ is to be determined.

\subsection{Wavelets}

In this subsection we will provide a brief account of wavelet transform and Multiresolution analysis (MRA). We first define the scaling function $\varphi(x)$ and the sequence $\left\{\alpha_{p}, p \in Z\right\}$ such that

$$
\varphi(x)=\sum_{p} \alpha_{p} \varphi\left(2^{j} x-p\right)
$$

By using this dilation and translation [4], we defined a nested sequence spaces $\left\{V_{j}, j \in Z\right\}$ which is called MRA of $L^{2}(R)$ with the following properties

$$
V_{j} \subset V_{j+1}, j \in Z
$$

$$
\begin{aligned}
& V_{-\infty}=\bigcap_{j \in Z} V_{j}=\{0\} \\
& \bigcup_{j} V_{j} \text { is dense in } L^{2}(R) \\
& \varphi(x) \in V_{j} \Leftrightarrow \phi(2 x) \in V_{j+1}
\end{aligned}
$$

For the subspace $V_{1}$ is built by $\varphi(2 x-p), \quad p \in Z$ then $V_{0}=\{\varphi(x-p), p \in Z\}$, and since $V_{0} \subset V_{1}$ we can write

$$
\phi(x)=\sum_{p} \alpha_{p} \phi(2 x-p) .
$$

In general,

$$
\phi(x)=\sum_{p} \alpha_{p} \phi\left(2^{j} x-p\right)=\sum_{p} \alpha_{p} \phi_{p, j}
$$

Any function $f(x) \in L^{2}(R)$ can be approximated by scaling functions in one of the subspace in the given nested sequence. In fact, for each $j$ we define the orthogonal complement subspace $W_{j}$ of $V_{j}$ in the subspace $V_{j+1}$. The orthogonal basis of $W_{j}$ is denoted by

$$
\psi_{j, p}(x)=\psi\left(2^{j} x-p\right),
$$

and the wavelet function can be obtained by

$$
\psi(x)=\sum_{p} \beta_{p} \phi_{j, p}(x) .
$$

Some interesting properties of scaling and wavelet functions make wavelet method more efficiently than quadrature formula methods and spline approximations in solving Integral equations. A lot of computational time and storage capacity can be saved since we do not require a huge number of arithmetic operations partly due 
to the following properties.

1) Vanishing Moments:

$$
\int_{-\infty}^{\infty} x^{k} \psi(x) \mathrm{d} x=0, k=0, \cdots, m-1 .
$$

and in this case the wavelet is said to have a vanishing moments of order $\mathrm{m}$,

2) Semiorthogonality:

$$
\begin{aligned}
& \left\langle\psi_{j, p}(x), \psi_{j, k}(x)\right\rangle=\int_{-\infty}^{\infty} \psi_{j, p}(x) \psi_{j, k}=0 ; \\
& p \neq k ; j, k, p \in Z
\end{aligned}
$$

The set of scaling functions $\left\{\varphi_{n, .}\right\}$ are orthogonal at the same level n, which means:

$$
\begin{aligned}
& \left\langle\phi_{n, k}(x) \phi_{n, p}(x)\right\rangle=\int_{-\infty}^{\infty} \phi_{n, k}(x) \phi_{n, p}(x) \mathrm{d} x=\delta_{k, p}, \\
& n, k, p \in Z
\end{aligned}
$$

Coiflet (of order L) has more symmetries and is an orthogonal multiresolution wavelet system with,

$$
\begin{gathered}
M_{k}=\int x^{k} \phi(x) \mathrm{d} x=0, k=1,2, \cdots, L-1 . \\
\int x^{k} \psi(x) \mathrm{d} x=0, k=0,1, \cdots L-1 .
\end{gathered}
$$

where $\left\{M_{k}\right\}$ are the moments of the scaling functions.

\subsection{Scaling Function Interpolation}

The function $f(x)$ can be interpolated by using the basis functions in the subspace $V_{j}$ as follows.

$$
f^{j}(x)=\sum_{p} a_{p} \phi\left(2^{j} x-p\right)
$$

where $a_{p}$ are the coefficients evaluated by using Equation (12) such that

$$
a_{p}=\left\langle f(x), \phi_{j, p}(x)\right\rangle=\int f(x) \phi\left(2^{j} x-p\right) \mathrm{d} x .
$$

Hence the Equation (15) becomes:

$$
f^{j}(x)=\sum_{p}\left(\int f(x) \phi\left(2^{j} x-p\right) \mathrm{d} x\right) \phi\left(2^{j} x-p\right) .
$$

On the other hand, one can use sampling values of $f$ at certain points to approximate the function $f$. It is proved in [5], namely, an interpolation theoremusing coiflet such that if $\phi(x)$ and $\psi(x)$ are sufficiently smooth and satisfy the Equations (10)-(14) and the function $f(x) \in C^{k}(\bar{\Omega})$, where $\Omega$ is a bounded open set in

$$
R^{2}, k \geq N \geq 2, j \in Z
$$

Then,

$$
\begin{aligned}
& f^{j}(x, y)=\frac{1}{2} \sum_{p, q \in \Lambda} f\left(\frac{p+c}{2^{j}}, \frac{q+c}{2^{j}}\right) \phi_{j, p}(x) \phi_{j, q}(y), \\
& (x, y) \in \Omega
\end{aligned}
$$

where the index set is

$$
\Lambda=\left\{(p, q) \mid\left(\sup \left(\phi_{j, p}\right) \otimes \sup \left(\phi_{j, q}\right)\right) \cap \Omega \neq \phi\right\},
$$

Sup denote the support of a function. In addition, the moment $M_{l}$ satisfies

$$
\begin{aligned}
& M_{l}=(c)^{l}, l=1,2, \cdots, N-1 . \\
& c=M_{1} .
\end{aligned}
$$

Then,

$$
\left\|f-f^{j}\right\|_{L^{2}(\Omega)} \leq C\left\|f^{(N)}\right\|_{\infty}\left(\frac{1}{2^{j}}\right)^{N} .
$$

where $C$ is a constant depending only on $N$, diameter of $\Omega$ and

$$
\left\|f^{(N)}\right\|_{\infty}:=\max _{(x, y) \in \Omega, m=0, \cdots, N}\left|\frac{\partial^{N} f}{\partial x^{m} \partial y^{N-m}}(x, y)\right| .
$$

For the function with one variable, we have

$$
f^{j}(x)=\frac{1}{2^{j}} \sum_{p} f\left(\frac{p}{2^{j}}\right) \phi_{j, p}(x), x \in[a, b],
$$

and

$$
\left|f-f^{j}\right|_{L^{2}[a, b]} \leq C\left\|f^{(N)}\right\|_{\infty}\left(\frac{1}{2}\right)^{N} .
$$

where

$$
\left\|f^{(N)}\right\|_{\infty}:=\max _{x \in(a, b), m=0, \cdots, N}\left|\frac{\partial^{N} f}{\partial x^{m}}(x)\right| .
$$

\section{Solve Fredholm Integral Equations Using Coiflet}

In this section we will apply coiflet and the interpolation formula (18) to solve the Fredholm integral Equation (1). The unknown function $y(x)$ in Equation (1) can be expanded in term of the scaling functions $\varphi_{j, p}(x)$ in the subspace $V_{j}$ such that

$$
y^{j}(x)=\sum_{p} a_{p} \phi_{j, p}(x) .
$$

Consider the Equation (1) and the function $y(x)$ which is defined on the interval $[a, b]$ and the scaling function $\phi(x)$ defined on the interval $(a, b)$ then we have the index:

$$
\Lambda=\left\{2^{j} a-d, 2^{j} a-d+1, \cdots, 2^{j} b-c\right\} .
$$

By applying Equation (21) into Equation (1), we get the system,

$$
\begin{aligned}
& \sum_{p \in \Lambda} a_{p} \phi\left(2^{j} x-p\right) \\
& =g(x)+\int_{a}^{b} k(x, t) \sum_{p} a_{p} \phi\left(2^{j} t-p\right) \mathrm{d} t,
\end{aligned}
$$

which is equivalent to the following system, 


$$
\sum_{p \in \Lambda} a_{p}\left(\phi\left(2^{j} x-p\right)-\int_{a}^{b} k(x, t) \phi\left(2^{j} t-p\right) \mathrm{d} t\right)=g(x),
$$

where thecoefficients $\left\{a_{p}, p \in \Lambda\right\}$ can be evaluated by substituting $\left\{x_{p} \in[a, b], p \in \Lambda\right\}$ into the system (23). Moreover, the system (23) can be expressed in compact form,

$$
A(B-C)=G
$$

where

$$
\begin{aligned}
& A=\left[a_{p}\right], B=\left[\phi\left(2^{j} x_{p}-p\right)\right], \\
& C=\int_{a}^{b} k\left(x_{p}, t\right) \phi\left(2^{j} t-p\right) \mathrm{d} t, G=\left[g\left(x_{p}\right)\right] .
\end{aligned}
$$

Then $A=G(B-C)^{-1}$.

This gives rise to coefficients in (21) and we obtain a numerical solution of (1). In what follows, we will derive a convergence theorem of this numerical solution.

\section{Error Analysis}

In this section will discuss the convergence rate of our method for solving linear Fredholm integral Equation (1).

Theorem 1. In Equation (1), supposethat the function $k(x, t) \in C([c, d] \times[a, b])$, and the functions $g(x)$ and $y(x) \in C[a, b]$, for $j \in Z$,

$$
y^{j}(x)=\sum_{p} a_{p} \phi\left(2^{j} x-p\right)
$$

is an approximate solution of the Equation (1) with the coefficients obtained in (24). Then,

$$
\|e(x)\|=\left\|y(x)-y^{j}(x)\right\| \leq C\left(\frac{1}{2}\right)^{j}
$$

where,

$$
\|e(x)\|=\left|\int_{a}^{b} e(x)\right| \cong \frac{1}{|\Lambda|} \sum_{p \in \Lambda} e\left(x_{p}\right) .
$$

Proof. Subtracting Equation (25) from Equation (1) and taking the norm for both sides, we get the following

$$
\begin{aligned}
\|e(x)\| & =\left\|\sum_{p} a_{p} \phi\left(2^{j} t-p\right)-y(x)\right\| \\
& =\left\|\int_{a}^{b}\left(k(x, t)\left(\sum_{p} a_{p} \phi\left(2^{j} t-p\right)-y(t)\right)\right) \mathrm{d} t\right\| \\
& \leq\left\|\int_{a}^{b} k(x, t) d t\right\|\left\|\int_{a}^{b}\left(\sum_{p} a_{p} \phi\left(2^{j} t-p\right)-y(t)\right) \mathrm{d} t\right\| \\
& =c_{1}\left\|\int_{a}^{b}\left(\sum_{p} a_{p} \phi\left(2^{j} t-p\right)-y(t)\right) \mathrm{d} t\right\|
\end{aligned}
$$

where $\quad c_{1}=\left\|\int_{a}^{b} k(x, t) \mathrm{d} t\right\|$.
By [5], the unknown function $y(x)$ can be interpolated by using coiflet such that:

$$
y^{j}(x)=\sum_{p} y\left(\frac{p}{2^{j}}\right) \phi\left(2^{j} x-p\right) .
$$

Let $t=x$ in Equation (28) then add and subtract it in Equation (27), we get the following inequalities.

$$
\begin{aligned}
& \|e(x)\| \leq c_{1} \| \int_{a}^{b}\left(\sum_{p} a_{p} \varphi\left(2^{j} t-p\right)-y(t)\right. \\
& \left.+\sum_{p} y\left(\frac{p}{2^{j}}\right) \phi\left(2^{j} t-p\right)-\sum_{p} y\left(\frac{p}{2^{j}}\right) \phi\left(2^{j} t-p\right)\right) \mathrm{d} t \| \\
& \leq c_{1}\left(\left\|\int_{a}^{b}\left(\sum_{p} y\left(\frac{p}{2^{j}}\right) \varphi\left(2^{j} t-p\right)-y(t)\right) \mathrm{d} t\right\|\right. \\
& \left.+\left\|\int_{a}^{b} \sum_{p} y\left(\frac{p}{2^{j}}\right) \phi\left(2^{j} t-p\right)-\sum_{p} a_{p} \phi\left(2^{j} t-p\right) \mathrm{d} t\right\|\right)
\end{aligned}
$$

which equals to the equation

$$
\begin{aligned}
& C_{1}\left(\left\|\int_{a}^{b} y\left(\frac{p}{2^{j}}\right) \phi\left(2^{j} t-p\right)-y(t) \mathrm{d} t\right\|\right. \\
& \left.+\left\|\sum_{p}\left(y\left(\frac{p}{2^{j}}\right)-a_{p}\right) \int_{a}^{b} \phi\left(2^{j} t-p\right) \mathrm{d} t\right\|\right) .
\end{aligned}
$$

By [5], we have

$$
\sum_{p} y\left(\frac{p}{2^{j}}\right) \phi\left(2^{j} t-p\right)-y(t) \leq c_{0}\left\|f^{(N)}\right\|_{\infty}\left(\frac{1}{2^{j}}\right)^{N} .
$$

Since $\sum_{p}\left(y\left(\frac{p}{2^{j}}\right)-a_{p}\right)$ is finite we define it as

$$
\sum_{p}\left(y\left(\frac{p}{2^{j}}\right)-a_{p}\right)=c_{2} .
$$

Using the above results and the orthonomality of the scaling functions $\{\phi(x)\}$, we conclude that

$$
\|e(x)\| \leq c_{1}\left(c_{0}\left\|f^{(N)}\right\|\left(\frac{1}{2^{j}}\right)^{N}+c_{2}\left(\frac{1}{2}\right)^{j}\right)=c\left(\frac{1}{2}\right)^{j} .
$$

\section{Numerical Examples}

In the following examples, we will solve linear Fredholm integral Equation (1) using coiflet of order 5 and provide errors between exact solutions and our numerical solutions at different resolution levels. Both examples are also presented in [6] by using different method.

\section{Example 1.}

Consider $y(x)=g(x)+\int_{a}^{b} k(x, t) y(t) \mathrm{d} t$, where

$$
g(x)=\sin x-x, k(x, t)=x t .
$$


The exact solution is $y(x)=\sin (x)$ and $x \in\left[0, \frac{\pi}{2}\right]$

\section{Example 2.}

Consider

$$
y(x)=g(x)+\int_{a}^{b} k(x, t) y(t) \mathrm{d} t,
$$

where $g(x)$ and $k(x, t)$ are given on the interval $[0,1]$ such that,

$$
g(x)=e^{x}-\frac{e^{x+1}-1}{x+1}, k(x, t)=e^{x t}
$$

The exact solution is $y(x)=e^{x}$.

We use our interpolation method to solve the above integral equations, and find the errors in Table 1.

\section{Conclusion}

In this work, we use our interpolation method by using coiflets to solve Fredholm integral equations, and compare our results with those in [6]. It turns out our method is more efficient with better accuracy. Moreover, our method can be applied to different kind of integral equations as well as integral-algebraic equations. Although the results in the above examples don't seem to have

Table 1. The error $e(x)$ for examples 1 and 2 with different values of $\boldsymbol{j}$.

\begin{tabular}{ccc}
\hline $\mathrm{j}$ & Example 1 & Example 2 \\
\hline 1 & $1.45478 \times 10^{-6}$ & $7.47811 \times 10^{-8}$ \\
2 & $9.32286 \times 10^{-8}$ & $9.94622 \times 10^{-8}$ \\
3 & $8.4068 \times 10^{-7}$ & $2.84008 \times 10^{-6}$ \\
4 & $4.87051 \times 10^{-7}$ & $1.12081 \times 10^{-6}$ \\
\hline
\end{tabular}

correlation with the level of resolutions but they basically validate our theorem. In fact, we can also interpolate the given functions in the integral equation. This would simplify the calculations in finding numerical solutions of integral equations. It would be interesting to use our method to solve nonlinear integral equations as well.

\section{Acknowledgements}

The authors would like to thank the anonymous referees for their helpful comments.

\section{REFERENCES}

[1] F. Brauer, "On a Nonlinear Integral Equation for Population Growth Problems," SIAM Journal on Mathematical Analysis, No. 6, 1975, pp. 312-317.

[2] F. Brauer and C. Castillo, "Mathematical Models in Population Biology and Epidemiology," Springer-Verlang, New York, 2001.

[3] T. A. Butorn, "Volterra Integral and Differential Equations," Academic Press, New York, 1983.

[4] K. C. Charles, "In Introduction to Wavelets," Academic Press, New York, 1992.

[5] E. B. Lin and X. Zhou, "Coiflet Interpolation and Approximate Solutions of Elliptic Partial Differential Equations," Numerical Methods for Partial Differential Equations, Vol. 13, No. 4, 1997, pp. 302-320. doi:10.1002/(SICI)1098-2426(199707)13:4<303::AID-N UM1>3.0.CO;2-P

[6] K. Maleknjaf and T. Lotfi, "Using Wavelet For Numerical Solution of Fredholm Integral Equations," Proceedings of the World Congress on Engineering, London, 2-4 July 2007, pp. 2-6. 